\section{SAT0626-HPR EXPLORING THE RELATION BETWEEN PAIN AND ACTIVITY AND PARTICIPATION BASED ON ICF IN CHILDREN AND ADOLESCENTS WITH JUVENIL IDIOPATIK ARTRIT: A PILOT STUDY}

E. Gur Kabul ${ }^{1}$, B. Basakci Calik ${ }^{1}$, B. Akar ${ }^{1}$, M. Bali ${ }^{1}$, Z. Ekici Tekin ${ }^{2}$, G. Otar Yener $^{2}$, S. Yuksel ${ }^{2}{ }^{1}$ Pamukkale University, School of Physical Therapy and Rehabilitation, Denizli, Turkey; ${ }^{2}$ Medical Faculty of Pamukkale University, Department of Pediatric Rheumatology, Denizli, Turkey

Background: Functional limitation and inactivation are the most important problems in children with Juvenil Idiopatik Artrit (JIA).

Objectives: The aim of this study was to link and allocate items of Childhood Health Assessment Questionnaire (CHAQ) with activity and participation based on International Classification of Functioning, Disability and Health (ICF). The other aim was to examine the relationship between the pain and activity and participation determined on the basis of CHAQ with Juvenil Idiopatik Artrit (JIA).

Methods: Thirty-seven children and adolescents (26 girls, 11 boys, mean age $=11.75 \pm 4.04$ years) were included. The mean BMI of the participants was $19.61 \pm 4.52 \mathrm{~kg} / \mathrm{cm}^{2}$. Inclusion criteria: To be diagnosed with JIA according to ILAR classification. Being in the 6-18 age range. To be stable in drug use for at least 3 months or longer. Exclusion criteria:The presence of another disease. Intraarticularsteroid injection or surgery in any joint in last 3 months. Evaluations were made by the same pediatric rheumatologist and physiotherapist (PT) by face to face interview method. Pain was evaluated by use of Numeric Rating Scale (NRS) $(0=$ no, $10=$ worst) and disability by $C H A Q$. As CHAQ score increases, disability increases. CHAQ has 8 categories. The highest score for any question determines the score for that category. The items of $\mathrm{CHAQ}$ were linked with ICF codes and allocated with the ICF components by three PT. Original scoring of CHAQ allocated to ICF components was used in order to calculate scores of activity and participation in accordance with clinical data for $37 \mathrm{JIA}$. The 20th item in the "Reach" category is not included in the calculation as it contains the body function component of ICF. The data was analyzed using Pearson's correlation coefficient.

Results: Mean score of NRS was $3.52 \pm 2.34$. Mean activity and participation score of $\mathrm{CHAQ}$ was $0.51 \pm 0.58,0.70 \pm 1.10$, respectively. Based on expert distinction, activity and participation categories of ICF were covered 24 and 5 items of $\mathrm{CHAQ}$, respectively. Pain had moderate correlation with activity ( $r=0.595$; $p=0.002)$ and participation $(r=0.604 ; p=0.001)$ for $C H A Q$. Activity had high correlation with participation $(r=0.702 ; \mathrm{p}=0.000)$.

Conclusion: Pain in children and adolescents with JIA is an important parameter affecting activity and participation. Pain should be evaluated in all aspects due to the limitation of both activity and participation. CHAQ largely contains the activity component of ICF. The development of new questionnaire is needed to assess the participation of JIA children and adolescents in more detail.

References:

[1] Schanberg LE. Widespread pain in children: when is it pathologic? Arthritis Rheum 2003:48:2402-2405.

Table 1. Linking and allocating items of $\mathrm{CHAQ}$

\begin{tabular}{lcc}
\hline Items & ICF Code & $\begin{array}{c}\text { ICF } \\
\text { Component }\end{array}$ \\
\hline DRESSING \& GROOMING Dress, including tying shoelaces & & $\mathrm{d} 5402$ \\
and doing buttons? & & $\mathrm{A}$ \\
Shampoo his/her hair? & $\mathrm{d} 5100$ & $\mathrm{~A}$ \\
Remove socks? & $\mathrm{d} 5403$ & $\mathrm{~A}$ \\
Cut fingernails? & $\mathrm{d} 520$ & $\mathrm{~A}$ \\
ARISING Stand up from a low chair or floor? & $\mathrm{d} 4103$ & $\mathrm{~A}$ \\
Get in and out of bed or stand up in crib? & $\mathrm{d} 4100$ & $\mathrm{~A}$ \\
EATING Cut his /her own meat? & $\mathrm{d} 550$ & $\mathrm{~A}$ \\
Lift a cup or glass to mouth? & $\mathrm{d} 4402$ & $\mathrm{~A}$ \\
Open a new cereal box? & $\mathrm{d} 440$ & $\mathrm{~A}$ \\
WALKING Walk outdoors on flat ground? & $\mathrm{d} 450$ & $\mathrm{~A}$ \\
Climb up five steps? & $\mathrm{d} 4551$ & $\mathrm{~A}$ \\
HYGIENE Wash and dry entire body? & $\mathrm{d} 510$ & $\mathrm{~A}$ \\
Take a tub bath? & $\mathrm{dd} 410$ & $\mathrm{~A}$ \\
Get on and off the toilet or potty chair? & $\mathrm{d} 4103$ & $\mathrm{~A}$ \\
Brush teeth? & $\mathrm{d} 5201$ & $\mathrm{~A}$ \\
Comb/brush hair? & $\mathrm{d} 5202$ & $\mathrm{~A}$ \\
REACH Reach and get down a heavy object from just above & $\mathrm{d} 4452$ & $\mathrm{~A}$ \\
his/her head? & & \\
Bend down to pick up clothing or a piece of paper from the & $\mathrm{d} 4105$ & $\mathrm{~A}$ \\
floor? & & \\
Pull on a sweater over his/her head? & $\mathrm{d} 5400$ & $\mathrm{~A}$ \\
Turn neck to look back over shoulder? & $\mathrm{b} 7101$ & Body Function \\
GRIP Write or scribble with pen or pencil? & $\mathrm{d} 170$ & $\mathrm{~A}$ \\
Open car doors? & $\mathrm{d} 4450$ & $\mathrm{~A}$ \\
Open jars which have been previously opened? & $\mathrm{d} 4453$ & $\mathrm{~A}$ \\
Turn faucets on and off? & $\mathrm{d} 4402$ & $\mathrm{~A}$ \\
& &
\end{tabular}

Table 1. Linking and allocating items of $\mathrm{CHAQ}$

\begin{tabular}{lcc}
\hline Items & ICF Code & $\begin{array}{c}\text { ICF } \\
\text { Component }\end{array}$ \\
\hline Push open a door when he/she to turn a door knob? & $\mathrm{d} 4453$ & $\mathrm{~A}$ \\
ACTIVITIES Run errands and shop? & $\mathrm{d} 6200$ & $\mathrm{P}$ \\
Get in and out of car or toy car or school? & $\mathrm{d} 4702$ & $\mathrm{P}$ \\
Ride bike or tricycle? & $\mathrm{d} 4750$ & $\mathrm{P}$ \\
Do household chores & $\mathrm{d} 6402$ & $\mathrm{P}$ \\
Run and play? & $\mathrm{d} 9201$ & $\mathrm{P}$
\end{tabular}

A:Activity, P:Participation

Disclosure of Interests: None declared

DOI: 10.1136/annrheumdis-2020-eular.2816

\section{SAT0627-HPR SEXUAL QUALITY OF LIFE IN 39 FEMALE PATIENTS WITH IDIOPATHIC INFLAMMATORY MYOPATHIES}

B. Heřmánková ${ }^{1,2}$, M. Špiritović ${ }^{1,2}, \mathrm{~S}$. Oreska ${ }^{2,3}$, H. Štorkánová ${ }^{2,3}$, M. Klein ${ }^{2,3}$, K. Pavelka ${ }^{2,3}$, L. Šenolt ${ }^{2,3}$, H. Mann ${ }^{2,3}$, J. Vencovský ${ }^{2,3}$, M. Tomčík ${ }^{2,3}$. ${ }^{1}$ Faculty of Physical Education and Sport, Charles University, Department of Physiotherapy, Prague, Czech Republic; ${ }^{2}$ Institute of Rheumatology, Prague, Czech Republic;

${ }^{3} 1$ st Faculty of Medicine, Charles University, Department of Rheumatology, Prague, Czech Republic

Background: Symptoms related to idiopathic inflammatory myopathies (IIM) such as weakness of skeletal muscles, pulmonary and articular involvement may have a negative impact on all aspects of life including sexual life.

Objectives: To assess sexual functioning in female IIM patients compared to age-/sex-matched healthy controls $(\mathrm{HC})$ and to analyze the potential impact of clinical features on sexual health.

Methods: In total, 39 women (29 currently have a partner) with IIM [mean age: 54.7, disease duration: 11.8 years, dermatomyositis (DM, 19)/ polymyositis (PM 16)/ necrotizing myopathy (IMNM, 3)/ inclusion body myositis (IBM, 1)] and 39 healthy controls (30 currently have a partner, mean age: 54.7 years) without rheumatic diseases filled in 11 well-established and validated questionnaires assessing sexual function (FSFI, SFQ28, BISFW), quality of sexual life (SQLL-F), pelvic floor function (PISQ-12, PFIQ-7), fatigue (FIS, MAF), physical activity (HAP), and depression (BDI-II). A standard laboratory testing was performed. Data are presented as mean \pm SEM.

Results: Patients with IIM reported significantly higher prevalence and greater severity of sexual dysfunction (FSFI, BISF-W, SFQ28) and worse sexual quality of life (SQoL-F) compared to $\mathrm{HC}$ (table 1). Worse scores in IIM patients were associated with increased inflammation [CRP: FSFI ( $r=-0.378$, $p=0.0190)$, SFQ-28 Satisfaction domain $(r=-0.346, p=0.0356)$, SQoL-F $(r=-$ $0.331, p=0,0479]$, greater muscle weakness of $\mathrm{m}$. gluteus maximus/ $\mathrm{m}$. gluteus medius/ $\mathrm{m}$. iliopsoas [FSFI: $(r=0.426, p=0.0368),(r=0.370, p=0.0368)$, $(r=0.394, p=0.0252)$, SQoL-F $(r=0.504, p=0.0044), \quad(r=0.421, p=0.0204)$, $(r=0.462, p=0.0100)$ ], greater fatigue [FIS: FSFI $(r=-0.358, p=0.0154)$, BISF-W $(r=-0.415, p=0.0084)$, SQoL-F $(r=-0.327, p=0.0481)]$, more severe depression [BDI-II: FSFI Arousal domain $(r=-0.357, p=0.0299)]$, deteriorated quality of life [HAQ: BISF-W $(r=-0.464, p=0.0033)$ ], and worse ability to perform physical activities [HAP: FSFI $(r=0.405, p=0.0105)$, BISF-W $(r=0.480, p=0.0019)]$. No associations were found with disease duration, prednisone dose or serum levels of muscle enzymes.

Table 1.

\begin{tabular}{|c|c|c|c|}
\hline Questionnaire: score range (meaning) & IIM (n=39) & $\mathrm{HC}(\mathrm{n}=39)$ & p-value \\
\hline FSFI: Female Sexual Function Index: 2(worst)-36(best) & $15.7 \pm 2.1$ & $20.7 \pm 2.0$ & $\mathrm{p}=0.0421$ \\
\hline $\begin{array}{l}\text { BISF-W: Brief Index of Sexual Function for Women: }-16 \\
\text { (worst)-75(best) }\end{array}$ & $14.6 \pm 2.8$ & $24.5 \pm 3.0$ & $p=0.0134$ \\
\hline $\begin{array}{l}\text { SFQ28 Desire domain: Sexual Function } \\
\text { Questionnaire: } 5 \text { (worst)-31(best) }\end{array}$ & $11.7 \pm 1.0$ & $14.7 \pm 1.0$ & $p=0.0457$ \\
\hline $\begin{array}{l}\text { PISQ-12: Pelvic Organ Prolapse/Urinary Incontinence } \\
\text { Sexual Questionnaire short form: } 0 \text { (best)-48(worst) }\end{array}$ & $14.9 \pm 0.8$ & $10.1 \pm 1.0$ & $p=0.0005$ \\
\hline $\begin{array}{l}\text { SQoL-F: Sexual Quality of Life Questionnaire - } \\
\text { Female: } 0 \text { (worst)-100(best) }\end{array}$ & $31.8 \pm 5.1$ & $80.7 \pm 2.5$ & $\mathrm{p}<0.0001$ \\
\hline $\begin{array}{l}\text { PFIQ7: Pelvic Floor Distress Inventory } \\
\text { Questionnaire - short form 7: } \\
\text { 0(best)-300(worst) }\end{array}$ & $24.7 \pm 6.3$ & $10.1 \pm 2.7$ & $p=0.0820$ \\
\hline FIS: Fatigue Impact Scale: 0 (best)-160(worst) & $55.3 \pm 5.5$ & $33.2 \pm 4.3$ & $\mathrm{p}=0.0025$ \\
\hline $\begin{array}{l}\text { MAF: Multidimensional Assessment of Fatigue Scale: } \\
1 \text { (best)-50(worst) }\end{array}$ & $22.0 \pm 2.0$ & $15.7 \pm 1.4$ & $p=0.0021$ \\
\hline $\begin{array}{l}\text { BDI-II: Beck's Depression Inventory II: } \\
\text { 0(best)-63(worst) }\end{array}$ & $12.8 \pm 1.5$ & $6.6 \pm 0.9$ & $p=0.0013$ \\
\hline $\begin{array}{l}\text { HAP: Human Activity Profile - adjusted activity score: } \\
0 \text { (worst)-94(best) }\end{array}$ & $51.0 \pm 3.5$ & $80.2 \pm 1.6$ & $<0.0001$ \\
\hline $\begin{array}{l}\text { HAQ: Health Assessment Questionnaire: } \\
0 \text { (best)-3(worst) }\end{array}$ & $1.1 \pm 0.1$ & $0.1 \pm 0.1$ & $\mathrm{p}<0.0001$ \\
\hline
\end{tabular}

